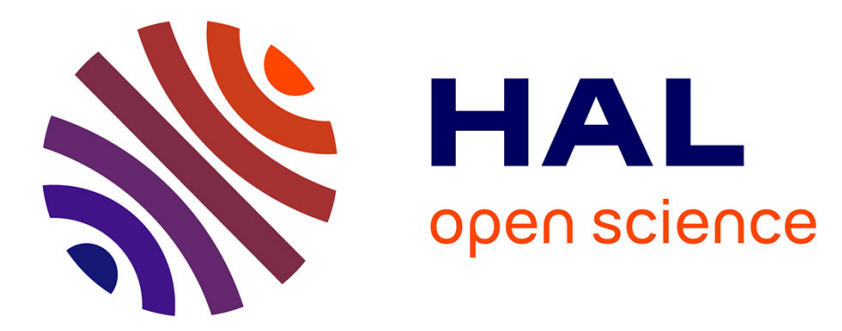

\title{
Curvature Discrimination for Dynamic Ultrasound Mid-Air Haptic Stimuli
}

Lendy Mulot, Guillaume Gicquel, William Frier, Maud Marchal, Claudio

Pacchierotti, Thomas Howard

\section{To cite this version:}

Lendy Mulot, Guillaume Gicquel, William Frier, Maud Marchal, Claudio Pacchierotti, et al.. Curvature Discrimination for Dynamic Ultrasound Mid-Air Haptic Stimuli. WHC 2021 - IEEE World Haptics Conference, Jul 2021, Montréal / Virtual, Canada. , pp.1. hal-03231609

\section{HAL Id: hal-03231609 \\ https://hal.inria.fr/hal-03231609}

Submitted on 20 May 2021

HAL is a multi-disciplinary open access archive for the deposit and dissemination of scientific research documents, whether they are published or not. The documents may come from teaching and research institutions in France or abroad, or from public or private research centers.
L'archive ouverte pluridisciplinaire HAL, est destinée au dépôt et à la diffusion de documents scientifiques de niveau recherche, publiés ou non, émanant des établissements d'enseignement et de recherche français ou étrangers, des laboratoires publics ou privés. 


\title{
Curvature Discrimination for Dynamic Ultrasound Mid-Air Haptic Stimuli
}

\author{
Lendy Mulot ${ }^{1}$, Guillaume Gicquel ${ }^{2}$, William Frier ${ }^{3}$, Maud Marchal ${ }^{4}$, Claudio Pacchierotti $^{2}$ and Thomas Howard ${ }^{2}$
}

\section{INTRODUCTION}

Ultrasound mid-air haptic interfaces are ideal for displaying tactile geometries [1], and can thus provide tangible sensations for 2D and 3D virtual shapes in human-computer interaction applications. Spatial and temporal sampling parameters, such as e.g. the number of points sampled on a shape, impact user's perception of a given shape [2]. The sampling strategy is thereafter defined as a combination of a spatial discretization of a shape and a set of rules for the temporal display order of the resulting points. This paper investigates the influence of sampling strategies on a user's ability to differentiate arc curvatures.

\section{USER STUDY ON CURVATURE DisCRIMINATION}

We focused our study on dynamic mid-air ultrasound tactile stimuli [1] displayed to a user's palm parallel to and centered $10 \mathrm{~cm}$ above an Ultraleap STRATOS Explore array. We aim to find the Just Noticeable Difference (JND) in curvature and its relation to the number of sampled points for simple sampling strategies. These were designed as a spatially uniform division of $n$ points displayed from the thumb towards the pinkie while dwelling on each point for $\frac{2}{n}$ seconds, and modulating the focal point intensity at $200 \mathrm{~Hz}$. The experiment was divided into five blocks, for $n=\{10$, $50,200,400,800\}$ points. Each block used the same four arc curvature radii $r$, ranging from a semi-circle to a flat line (see Fig. 1). We considered the flat line as an arc having the smallest radius to length ratio so that the height difference at the edges of the arc does not exceed the radius of a focal point. The stimuli were created using a custom software and the experiment was conducted using Psychopy [3]. Trials followed a 2-AFC protocol, with two stimuli displayed with

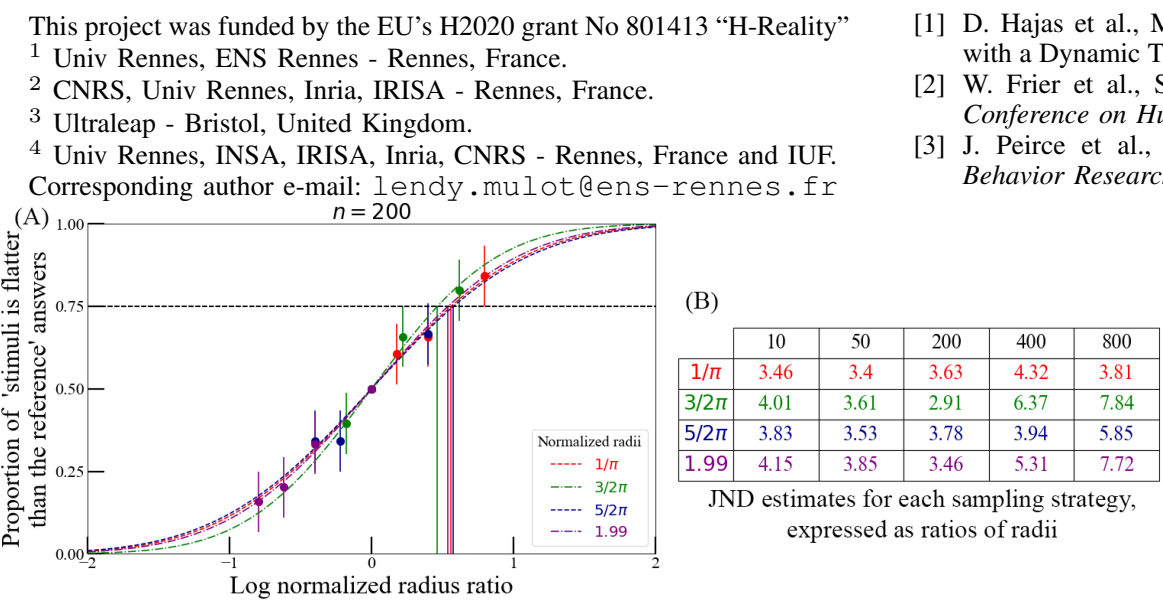

a $1500 \mathrm{~ms}$ break between them, followed by a request to identify the stimulus closest to a straight line. Each pair was displayed three times per block. Pair order within blocks block order were randomized for each user. We recruited 19 right-handed subjects ( 2 fem., 1 non-bin., 20-26 y.o.).

\section{RESULTS AND DISCUSSION}

Cumulative Gaussian curves centered on 0 (corresponding to a radius ratio of 1 ) were fitted to the mean response values for each stimulus-reference pair, yielding 4 estimates for JND in curvature, expressed as a radius ratio (see Fig. 1). JNDs ranged from 2.91 to 3.78 in the best performing sampling strategy $(n=200)$, and from 3.81 to 7.72 in the worst $(n=800)$, which may be explained by the greater difficulty for some subjects to perceive the stimuli for this sampling strategy. The presence of results exceeding $50 \%$ in every graph and the good fit of the psychometric curves show that curvature discrimination is possible using dynamic ultrasound haptic stimuli, regardless of the sampling strategy. Only stimuli at the limits of our range of radii yielded success rates above $75 \%$. The fact that discrimination never occurred with $100 \%$ accuracy indicates difficulties in perception of spatial features of ultrasound tactile stimuli in line with literature results (e.g. [1]).

As a next step, we will perform statistical analysis to determine whether sampling strategies have a significant effect on the curvature JND, and may conduct additional experiments to increase the JND estimates' accuracy. If a significant effect is found, we will attempt to replicate it and measure the curvature JND for static stimuli, to gain insight into optimal rendering parameters for ultrasound haptics.

\section{REFERENCES}

D. Hajas et al., Mid-Air Haptic Rendering of 2D Geometric Shapes with a Dynamic Tactile Pointer, IEEE Transactions on Haptics (2020). Human Factors in Computing Systems (2019).

J. Peirce et al., PsychoPy2: Experiments in behavior made easy,

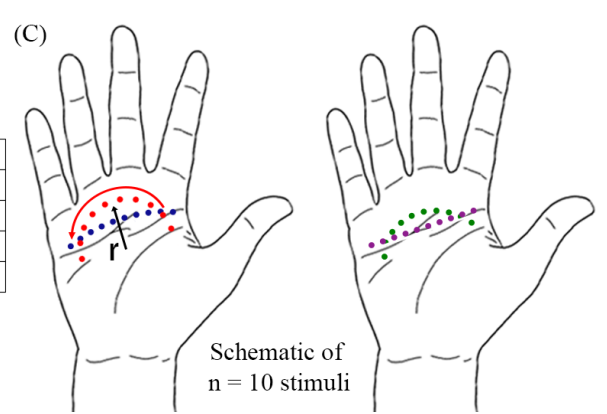

Fig. 1. (A) Example of mean proportion of "stimulus is flatter than the reference" answers as a function of the log stimulus to reference arc radius ratio, for the $n=200$ points sampling strategy. The red, green, blue and purple curves show the results for each of the reference radii in ascending order of value. Points and associated vertical lines show the mean answer proportion and associated 95\% confidence interval. (B) 75\% JND estimates for each radius (row) and strategy (col.). (C) $n=10$ stimuli schematic: the focal point moves from the thumb toward the pinkie, spending $200 \mathrm{~ms}$ on each point. 